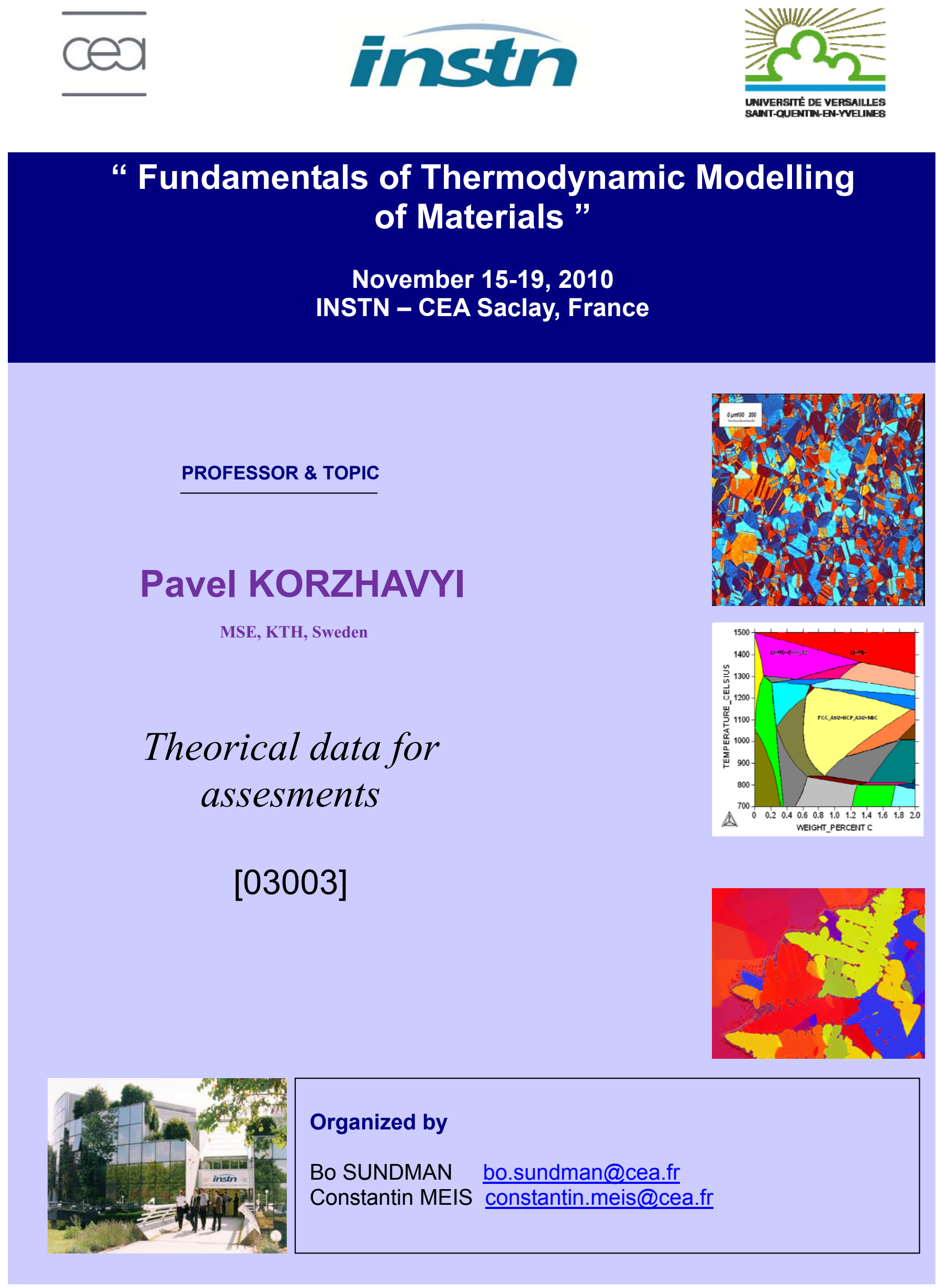

This is an Open Access article distributed under the terms of the Creative Commons Attribution-Noncommercial License 3.0, which permits unrestricted use, distribution, and reproduction in any noncommercial medium, provided the original work is properly cited. 


\section{Theoretical data for assessments}

\section{$0 y)$ XXX \\ Pavel A. Korzhavyi}

Applied Materials Physics

Department of Materials Science and Engineering

School of Industrial Engineering and Management

Royal Institute of Technology (KTH), Stockholm

\section{A modern CALPHAD flowchart}

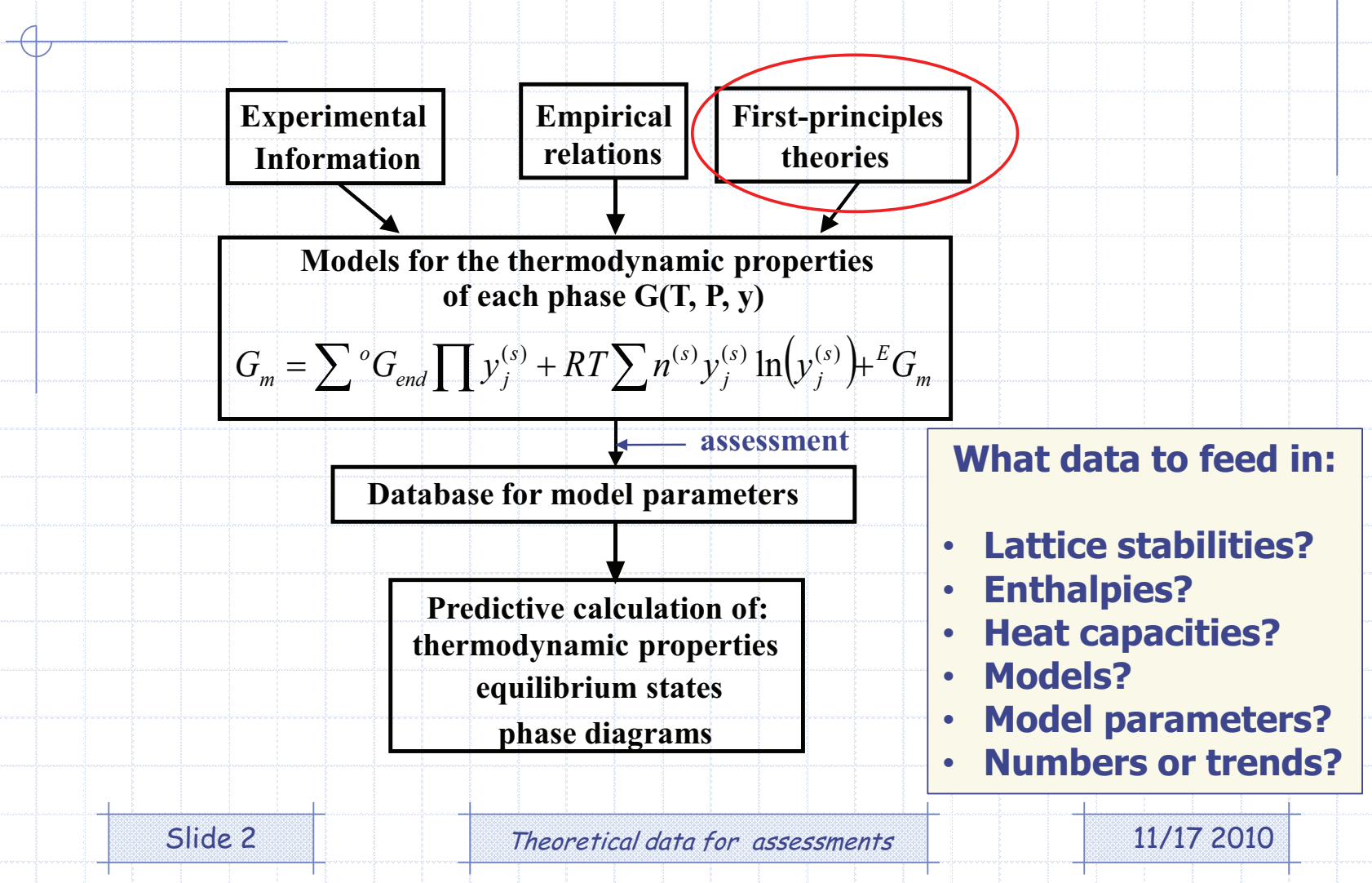




\section{Success example: NIST-JANAF data for diatomic gases}

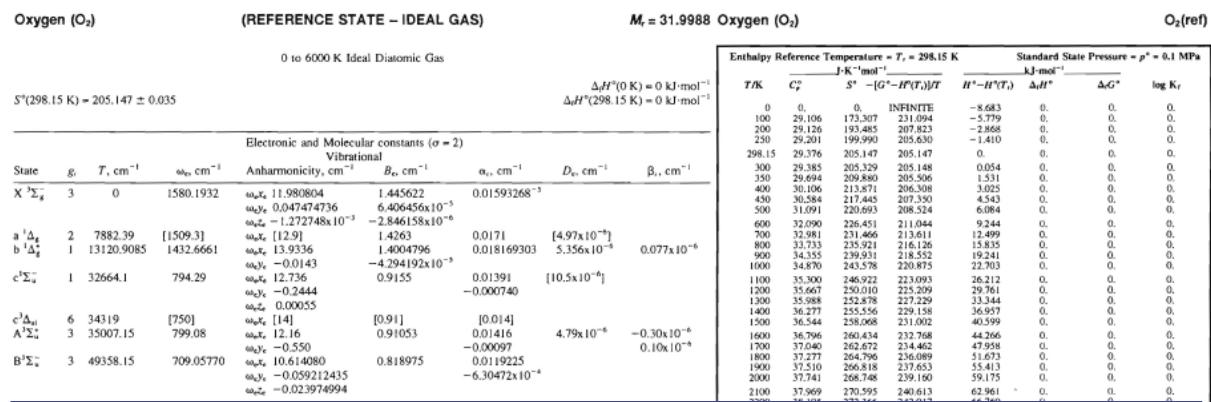

Internal degrees of freedom taken into account:

- Vibrational (harmonic and anharmonic)

- Rotational

- Rotation-vibration interactions

- Centrifugal stretching

- Electronic (transitions between molecular terms)

\section{$\mathrm{H}_{2}$ molecule}

Stable state: Paired spins, symmetric
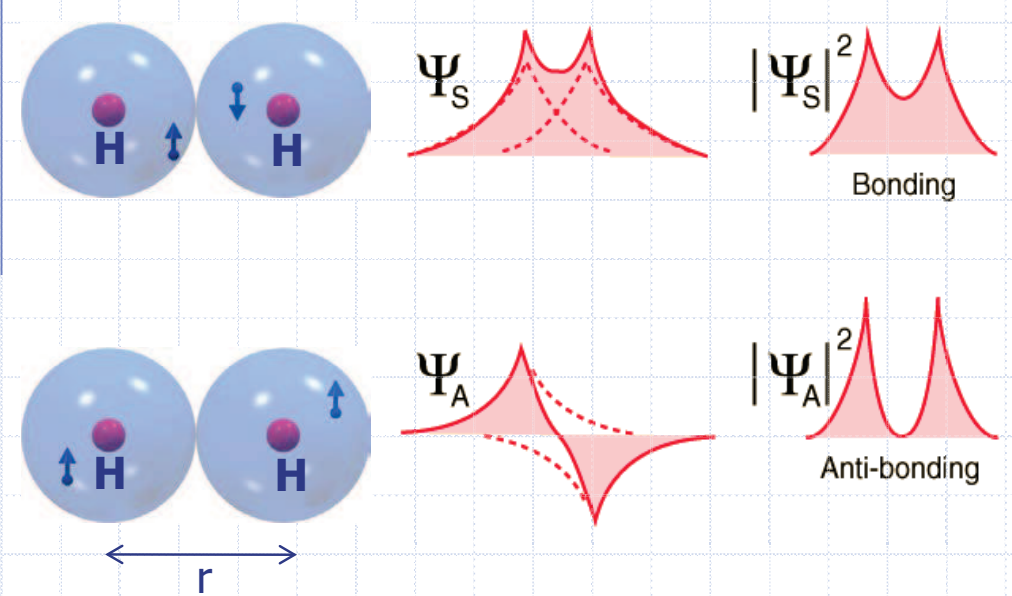

Unstable state: Unpaired spins, antisymmetric

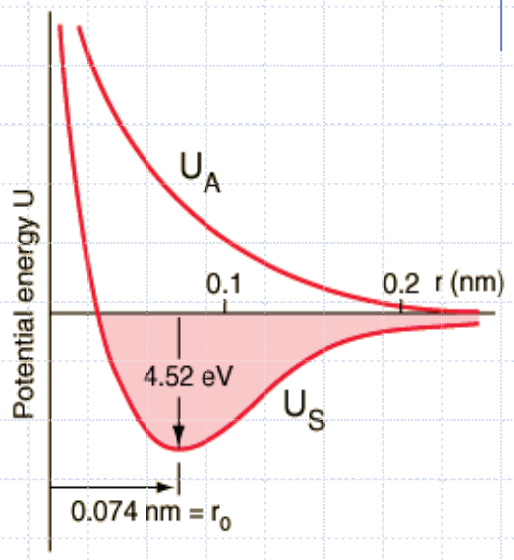




\section{Vibrational properties of dimer molecules}

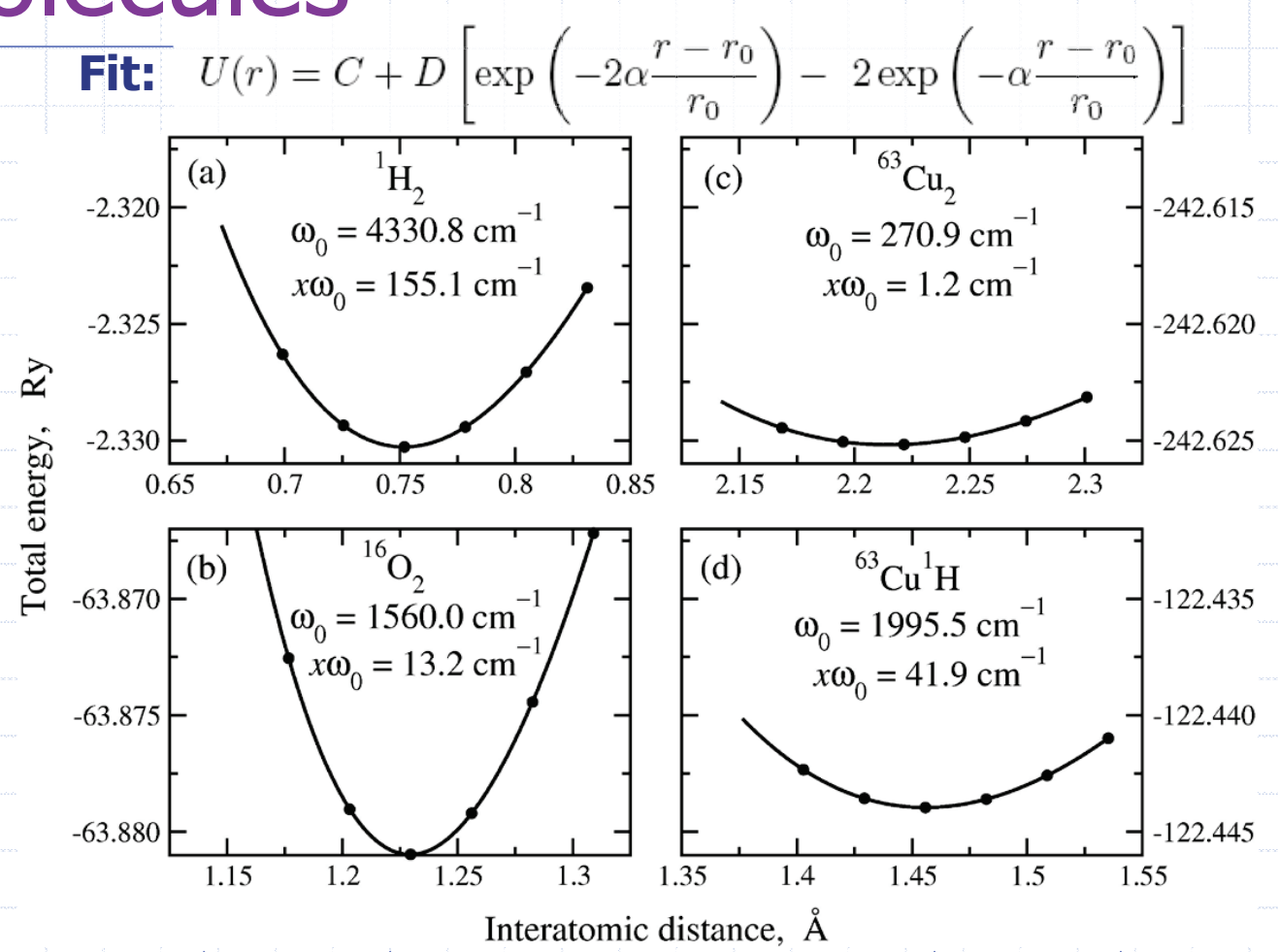

\section{Vibrational properties of dimer}

molecules

Spectrum: $E=C-D+\hbar \omega_{0}\left(n+\frac{1}{2}\right)-\hbar x \omega_{0}\left(n+\frac{1}{2}\right)^{2}$

Zero-point energy: $\quad \mathrm{ZPE} \approx \frac{1}{2} \hbar \omega_{0}-\frac{1}{4} \hbar x \omega_{0}$

\begin{tabular}{|c|l|r|r|r|r|}
\hline Dimer & Data source & \multicolumn{1}{|c|}{$r_{0}(\AA)$} & $\omega_{0}\left(\mathrm{~cm}^{-1}\right)$ & $x \omega_{0}\left(\mathrm{~cm}^{-1}\right)$ & $\mathrm{ZPE}\left(\mathrm{cm}^{-1}\right)$ \\
\hline${ }^{1} \mathrm{H}_{2}$ & This work & $\mathbf{0 . 7 5 1 7}$ & $\mathbf{4 3 3 0 . 7 6}$ & $\mathbf{1 5 5 . 1 4}$ & $\mathbf{2 1 2 6 . 5 9}$ \\
\hline & Exp. [1] & 0.7414 & 4401.21 & 121.34 & 2179.30 \\
\hline${ }^{16} \mathrm{O}_{2}$ & This work & $\mathbf{1 . 2 2 9 4}$ & $\mathbf{1 5 6 0 . 0 9}$ & $\mathbf{1 3 . 2 2}$ & $\mathbf{7 7 6 . 7 4}$ \\
\hline & Exp. [1] & 1.2075 & 1580.19 & 11.98 & 787.38 \\
\hline${ }^{63} \mathrm{Cu}_{2}$ & This work & $\mathbf{2 . 2 1 4 9}$ & $\mathbf{2 6 4 . 5 5}$ & $\mathbf{1 . 0 2}$ & $\mathbf{1 3 5 . 1 4}$ \\
\hline & Exp. [1] & 2.2197 & 270.89 & 1.21 & \\
\hline${ }^{63} \mathrm{Cu}^{1} \mathrm{H}$ & This work & $\mathbf{1 . 4 5 5 7}$ & $\mathbf{1 9 4 1 . 2 6}$ & $\mathbf{3 7 . 5 1}$ & $\mathbf{9 8 7 . 3 0}$ \\
\hline & Exp. [1] & 1.4626 & 1995.54 & 41.90 & \\
\hline
\end{tabular}

[1] K. K. Irikura, Experimental vibrational zero-point energies: Diatomic molecules , J. Phys. Chem. Ref. Data 36, 389 (2007). 


\section{Energy units and accuracy}

$1000 \mathrm{~cm}^{-1} \cong 0.124 \mathrm{eV} \cong 12.0 \mathrm{~kJ} / \mathrm{mol}$

Table 2: Absolute error (eV) of some density functional approximations in predicting the dissociation energy of small molecules [2].

\begin{tabular}{|c|c|c|c|c|}
\hline Molecule & LDA & GGA(PBE) & BLYP & PKZB \\
\hline $\mathrm{H}_{2}$ & +0.16 & -0.21 & -0.00 & +0.22 \\
\hline $\mathrm{O}_{2}$ & +2.36 & +1.01 & +0.64 & +0.47 \\
\hline $\mathrm{H}_{2} \mathrm{O}$ & +1.49 & +0.09 & +0.01 & -0.09 \\
\hline $\mathrm{N}_{2}$ & +1.69 & +0.64 & +0.49 & +0.03 \\
\hline
\end{tabular}

[2] S. Kurth, J.W. Perdew, and P. Blaha, Molecular and solid-state tests of density functional approximations: $L S D, G G A s$, and Meta-GGAs, Inlt. J.

Quantum Chem. 75, 899 (1999).

Slide 7

\section{Accuracy (continued)}

- Accuracy of DFT functionals is routinely checked in molecular and solid-state tests, whenever a new functional is created.

- It is good practice to find out the accuracy of various DFT approximations for your system.

\section{Some useful references:}

[2] S. Kurth, J.W. Perdew, and P. Blaha, Molecular and solid-state tests of density functional approximations: $L S D, G G A s$, and Meta-GGAs, Inlt. J. Quantum Chem. 75, 899 (1999).

[3] P. Haas, F. Tran, and P. Blaha, Calculation of the lattice constant of solids with semilocal functionals, Phys. Rev B 79, 085104 (2009).

[5] L. Pedroza, A.J.R. da Silva, K. Capelle, Gradient-dependent density functionals of the Perdew-Burke-Ernzerhof type for atoms, molecules, and solids, Phys. Rev. B 79, 201106 (2009).

[4] P. Haas, F Tran, P. Blaha, K. Schwarz, and R. Laskowski, Insight into the performance of GGA functionals for solid-state calculations, Phys. Rev. B 80, 195109 (2009).

[6] P.J. Feibelman, DFT Versus the "Real World" (or, Waiting for Godft), Top. Catal. 53, 417 (2010). 


\section{Phonon spectrum of $\mathrm{Cu}_{2} \mathrm{O}$}
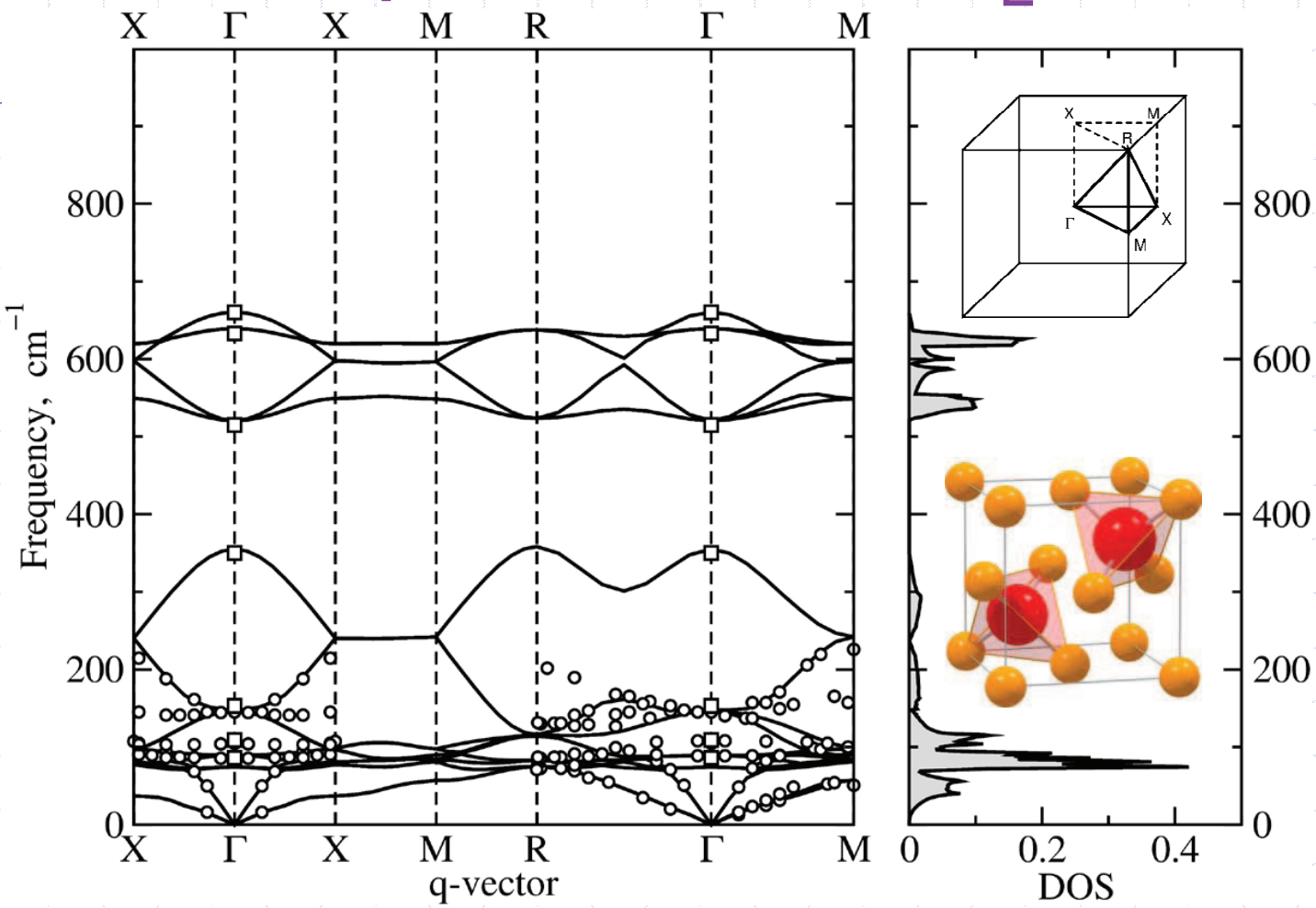

P.A. Korzhavyi and B. Johansson, Technical Report TR-10-30, (SKB, Stockholm, 2010).

\section{Thermodynamic properties of $\mathrm{Cu}_{2} \mathrm{O}$}

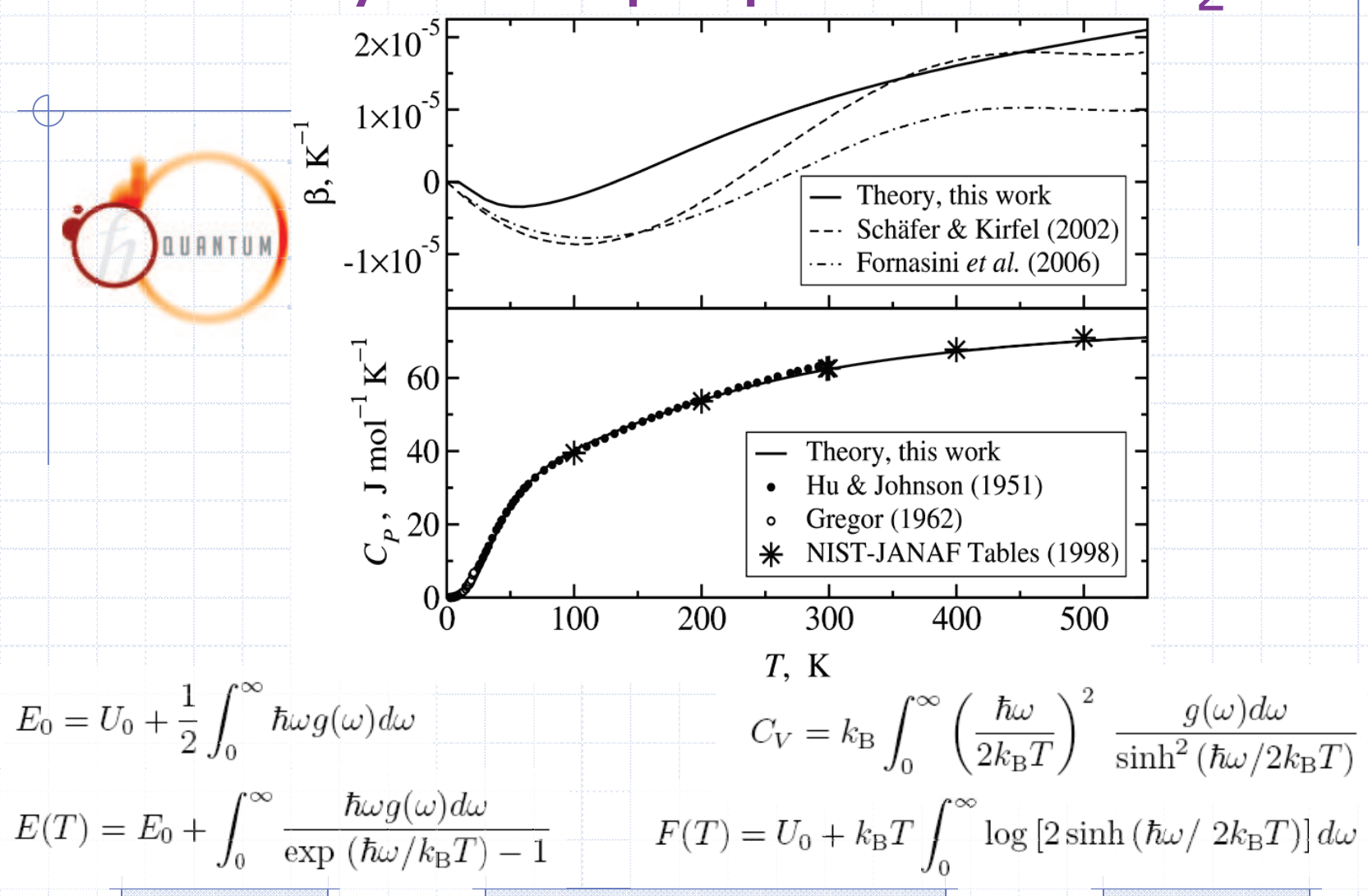




\section{Thermodynamic properties of $\mathrm{Cu}_{2} \mathrm{O}$}

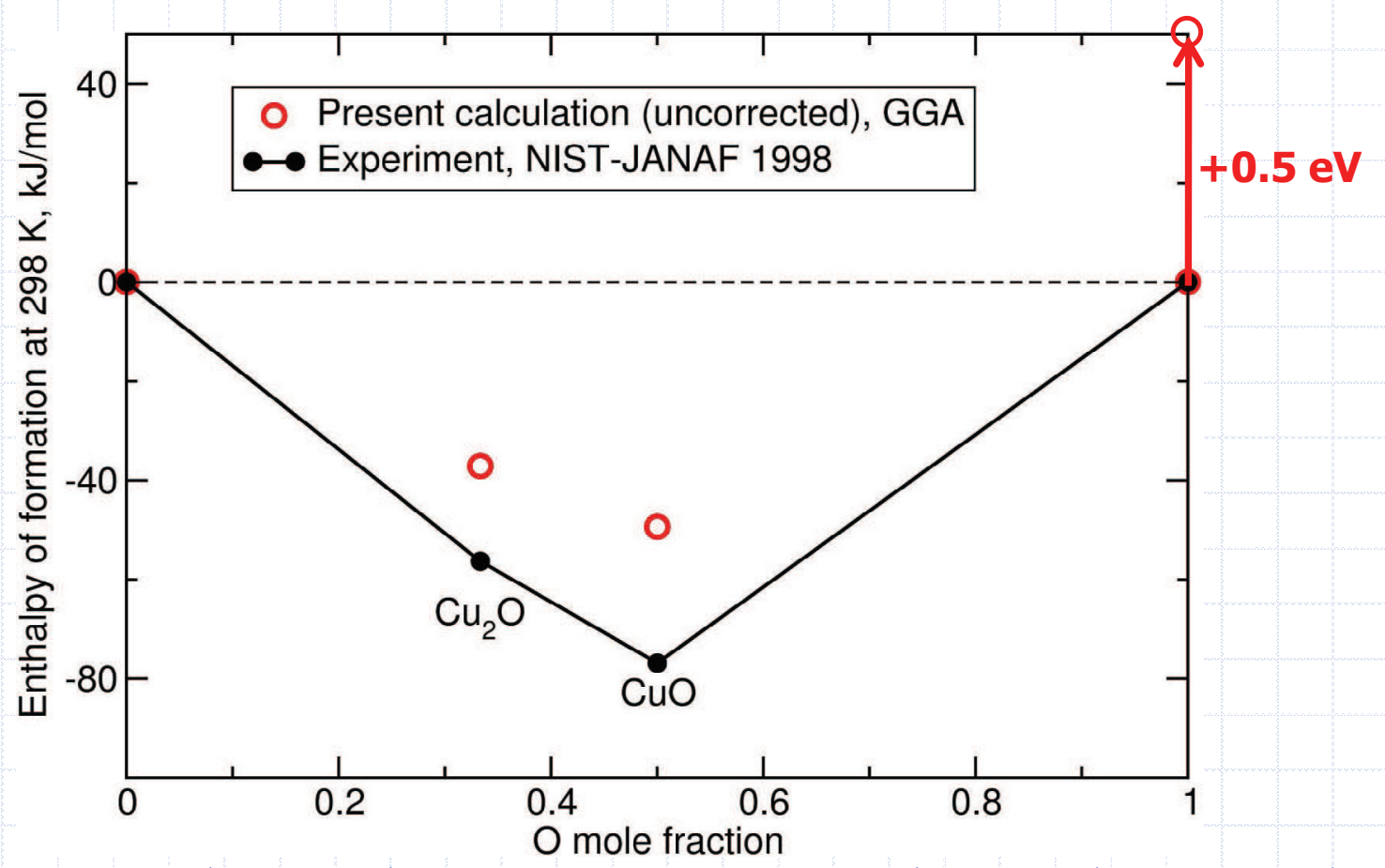

\section{Thermodynamic properties of $\mathrm{Cu}_{2} \mathrm{O}$}

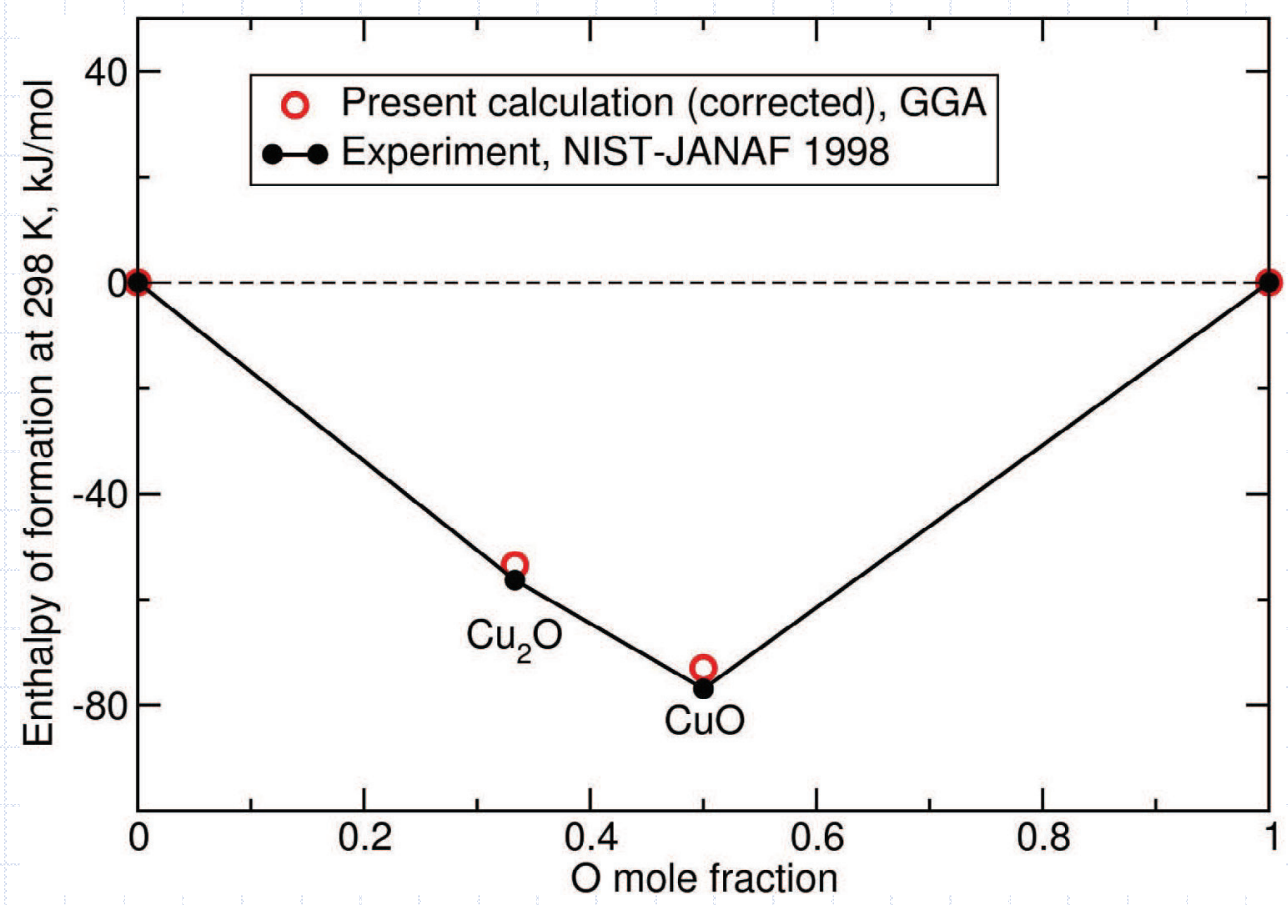




\section{Degrees of freedom to be taken into account in solids}

Time scale

- Atomic defects (non$>10^{-6} \mathrm{~s}$ stoichiometry, high temperature)

- Vibrational (harmonic and $\quad \sim 10^{-13} \mathrm{~s}$ anharmonic)

- Magnetic excitations

$\sim 10^{-14} \mathrm{~s}$

- Electronic excitations

$\sim 10^{-15} \mathrm{~s}$

\section{Compound energy formalism (CEF)}

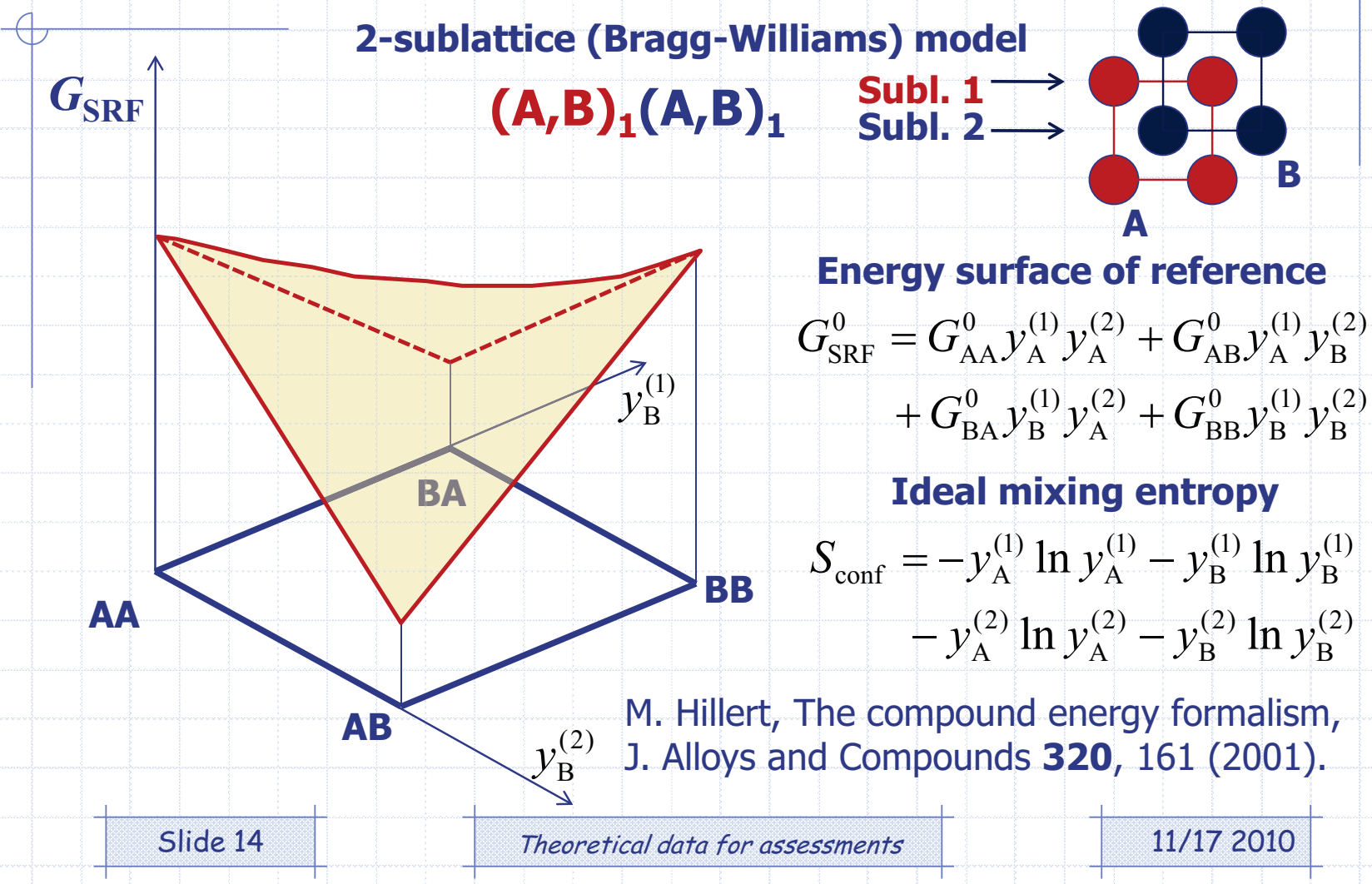




\section{4-sublattice model, bcc Fe-Ti: $2^{4}=16$ end members}

A2

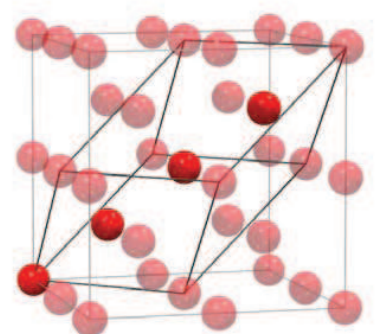

FFFF (1)

$\mathrm{DO}_{3}$

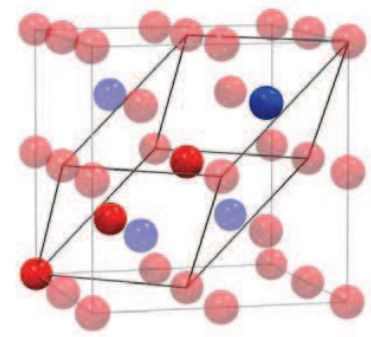

F F F T (4)

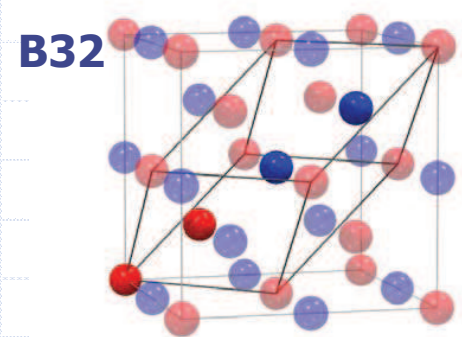

F F T T (4)

B2

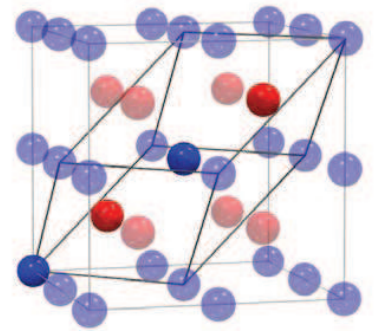

F T F T (2)
A2

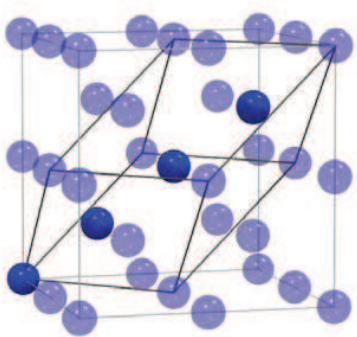

T T T (1)

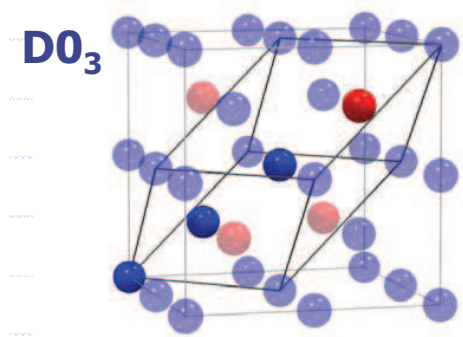

T T T F (4)

\section{4-sublattice model, bcc Fe-Ti:}

enthalpies of formation

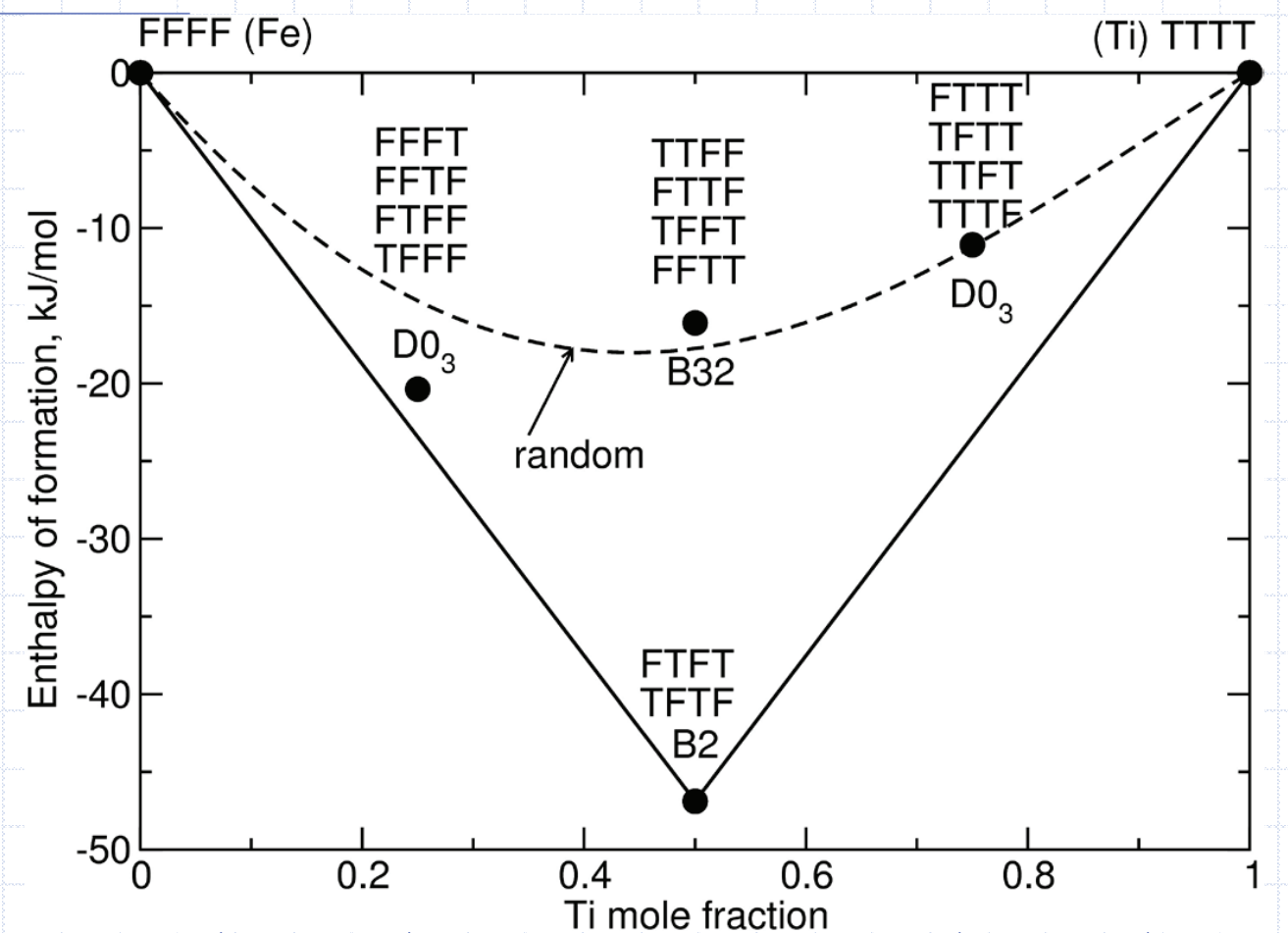




\section{A combined ab initio - CALPHAD study}
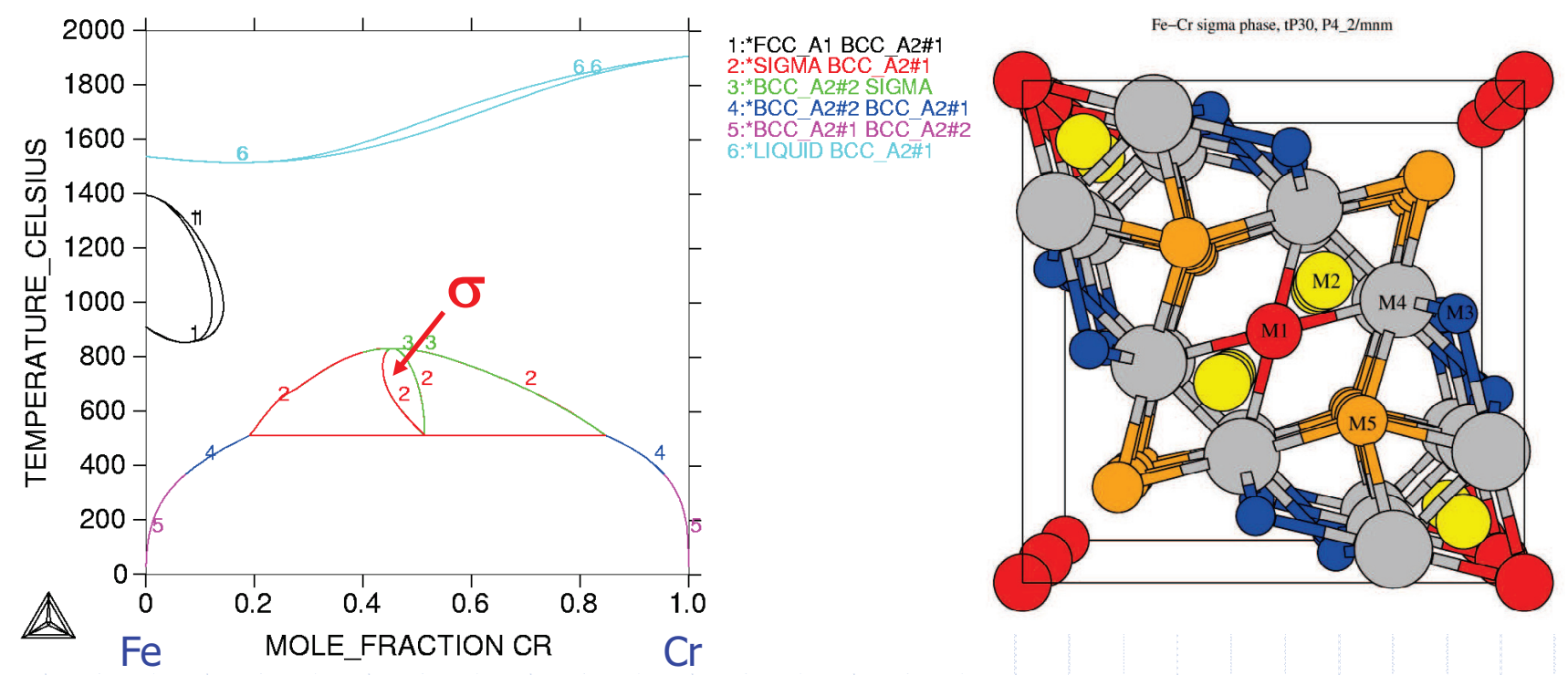

P.A. Korzhavyi, B. Sundman, M. Selleby, and B. Johansson, Atomic, electronic, and magnetic structure of iron-based sigma-phases, in Integrative and Interdisciplinary Aspects of Intermetallics, MRS Proc., 842, edited by M.J. Mills et al., (Warrendale, 2005), pp. S4.10.1-6.

\section{Slide 17 \\ Theoretical data for assessments \\ Crystallographically inequivalent sites}

$11 / 172010$

\section{Icosahedrally coordinated sites}

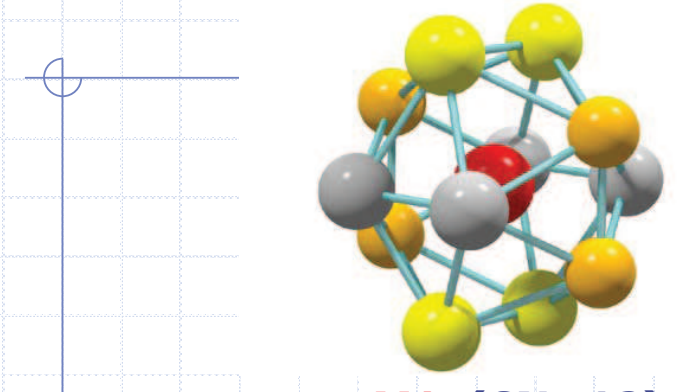

M1 $(\mathrm{CN}=12)$

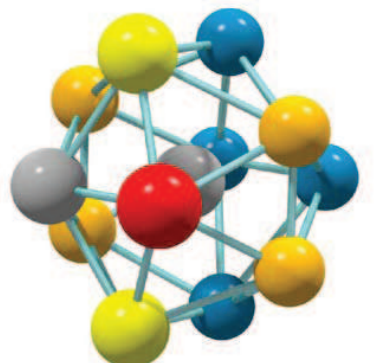

M4 ( $\mathrm{CN}=12)$

\section{High coordination number (high-CN) sites}

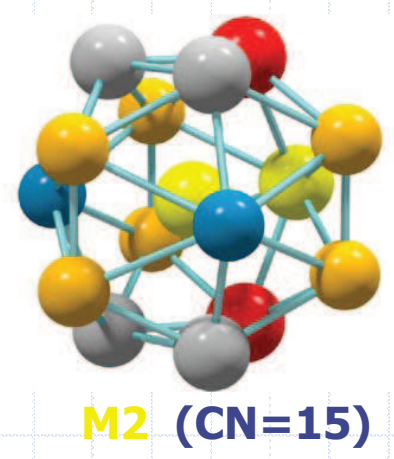

Slide 18

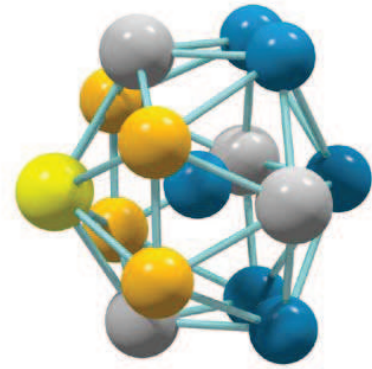

M3 $(\mathrm{CN}=14)$

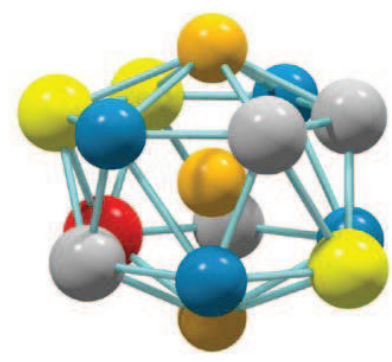

M5 $(\mathrm{CN}=14)$ 


\section{$\sigma$-phase model}

\section{$(\mathrm{Fe}, \mathrm{Cr})_{2}(\mathrm{Fe}, \mathrm{Cr})_{4}(\mathrm{Fe}, \mathrm{Cr})_{8}(\mathrm{Fe}, \mathrm{Cr})_{8}(\mathrm{Fe}, \mathrm{Cr})_{8}$}

$\rightarrow$ "end member compound": $\mathrm{FCCFC}=\mathrm{Fe}_{2} \mathrm{Cr}_{4} \mathrm{Cr}_{8} \mathrm{Fe}_{8} \mathrm{Cr}_{8}$

$\rightarrow 2^{5}=32$ end member compound energies.

These energies have been calculated from first principles

\section{Energies of the end-member compounds}

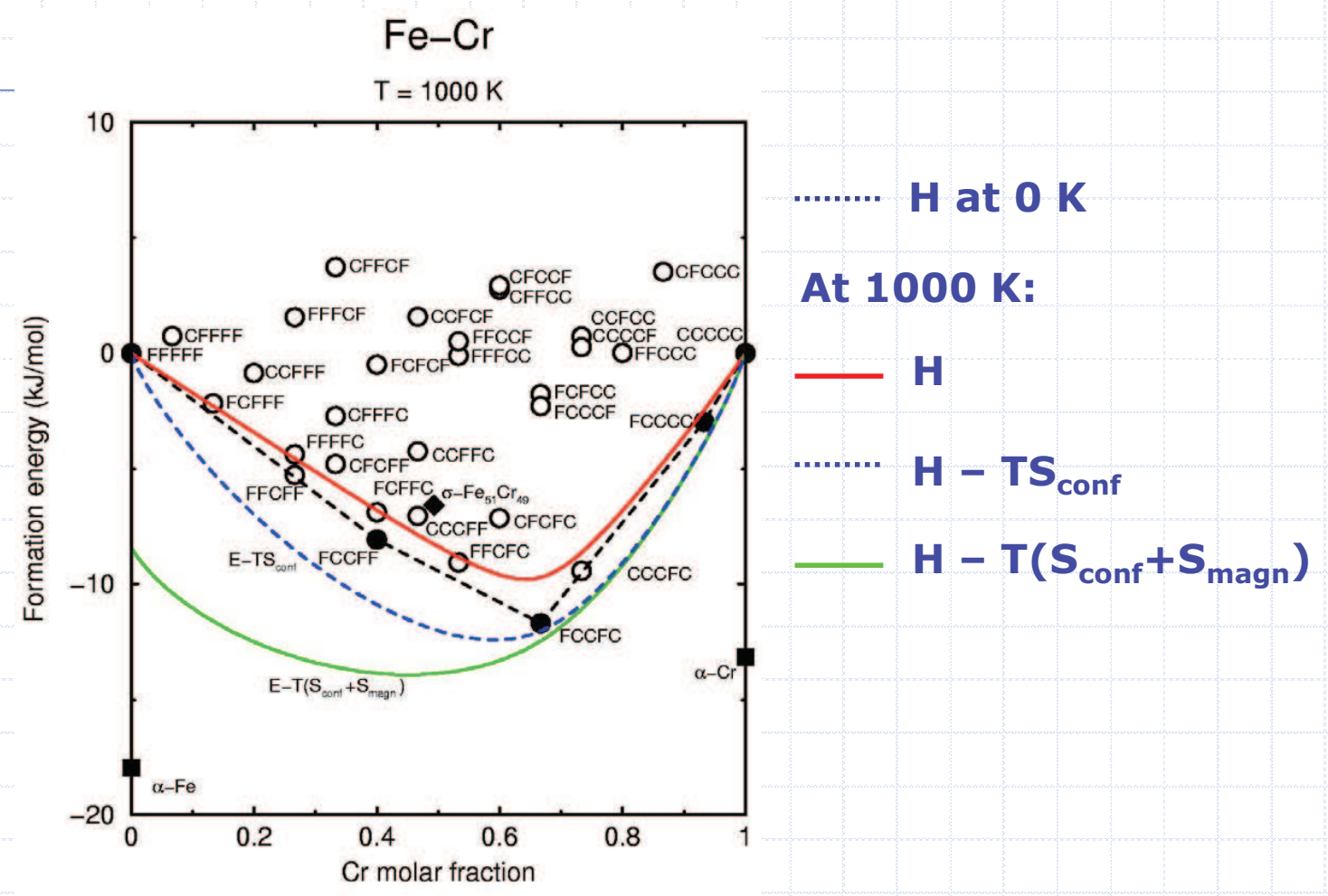




\section{Magnetic moments and entropy contribution}

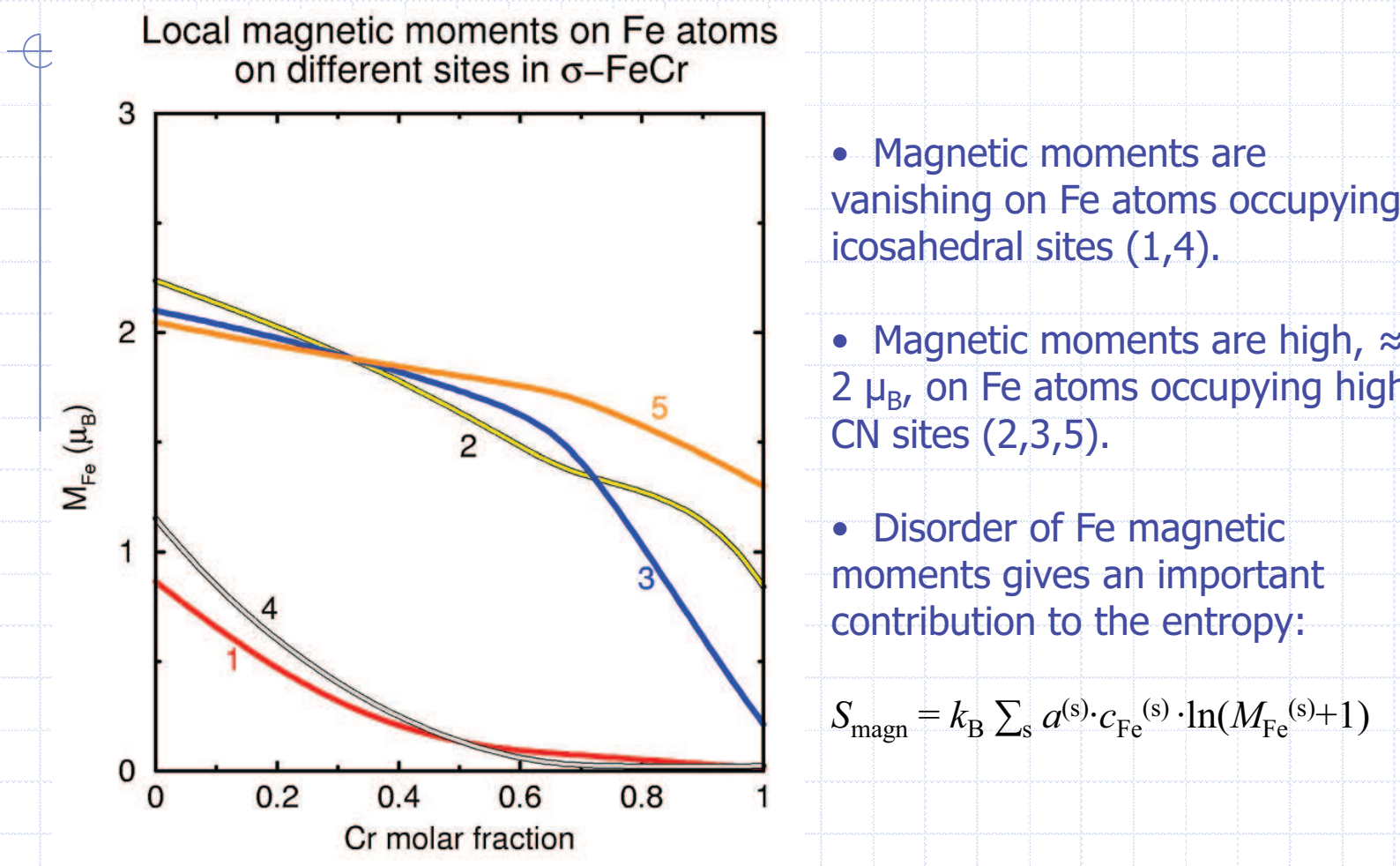

\section{Isoelectronic sigma phases}

\begin{tabular}{|c|c|c|c|c|c|c|c|c|}
\hline & $3 \mathrm{~B}$ & 4B & 5B & 6B & 7B & - & $-8 \mathrm{~B}$ & \\
\hline $3 \pi$ & 15 & $\frac{22}{\mathbf{T i}}$ & 2 & 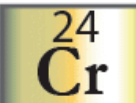 & Mn & $\mathrm{Fe}$ & Co & \\
\hline & & & 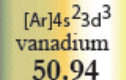 & 5 & 5.94 & $\begin{array}{l}\text { i } \\
550.80\end{array}$ & $\begin{array}{l}\text { cotodat } \\
5889\end{array}$ & . \\
\hline & & & & -42 & & 44 & 45 & \\
\hline 4d & & $\mathrm{Zr}$ & $\mathrm{Nb}$ & Mo & Tc & $\mathbf{R u}$ & Rh & Pd \\
\hline & 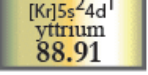 & 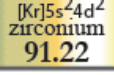 & 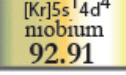 & 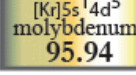 & 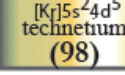 & $\begin{aligned} \text { ritionimum } \\
1010\end{aligned}$ & 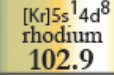 & 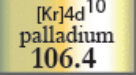 \\
\hline & 57 & & 7 & 74 & 7 & & 77 & \\
\hline & La* & Hf & $\mathrm{Ta}$ & W & $\operatorname{Re}$ & Os & Ir & $\mathrm{t}$ \\
\hline & anui & $\begin{array}{l}\text { 㘧位um } \\
178.5\end{array}$ & $\begin{array}{l}\text { 18tivitu } \\
\text { 180.9 }\end{array}$ & nistiten & 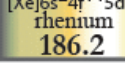 & $\begin{array}{l}\text { tam } \\
\text { o. }\end{array}$ & diuf & \\
\hline
\end{tabular}
$\mathbf{N}_{\mathrm{d}}=\mathbf{1}$
2
3
4
5
6
7
8 


\section{Mo-Ru sigma phase}

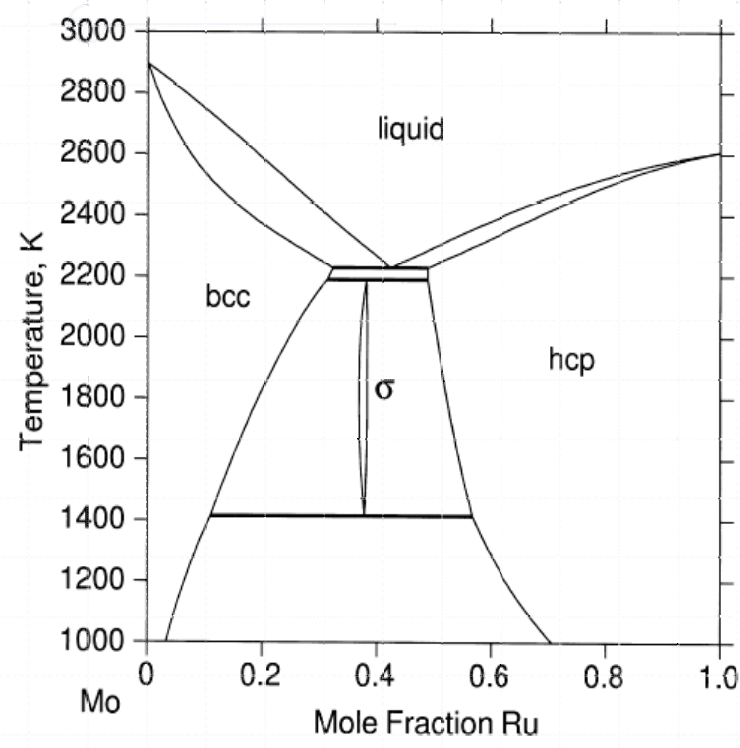

C.-S. Oh, H. Murakami, and H. Harada, J. Alloys Compounds 313, 115 (2000).

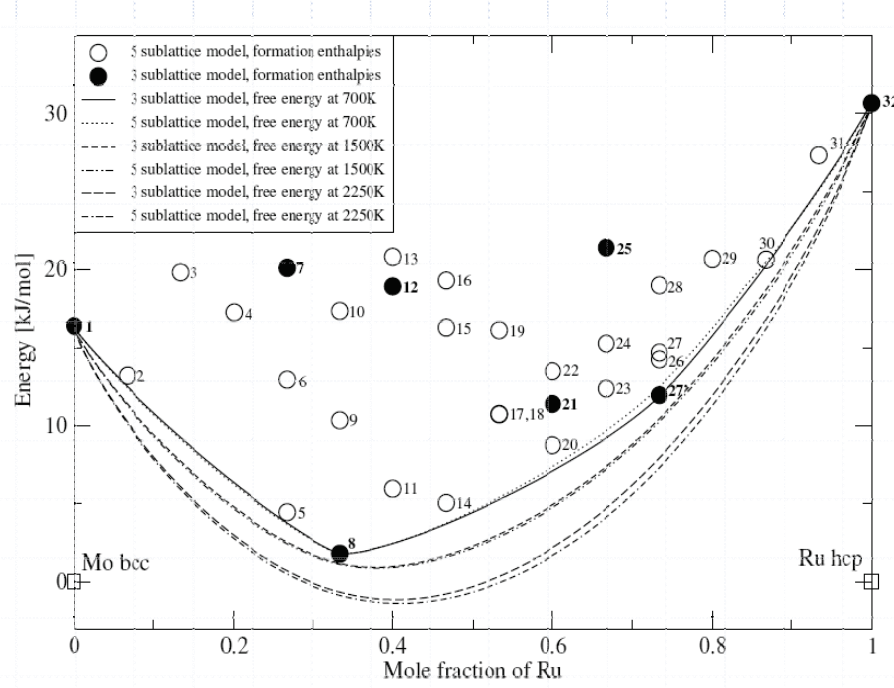

Fig. 1. Calculated enthalpies of formation for the end-member compounds of 5-sublattice model (open circles) and 3 sublattice model (filled circles). Free energies

O. Grånäs, P.A. Korzhavyi, A.E. Kissavos, and I.A. Abrikosov, CALPHAD 32, 171 (2008).

\section{Non-stoichiometry in titanium carbides and nitrides}

- $\quad \mathrm{TiC}_{\mathrm{x}}$ and $\mathrm{TiN}_{\mathrm{x}}$ crystallize in the B1 ( $\left.\mathrm{NaCl}\right)$ structure.

- Vacancies only on the non-metal sublattice, (Ti)(X,Va).

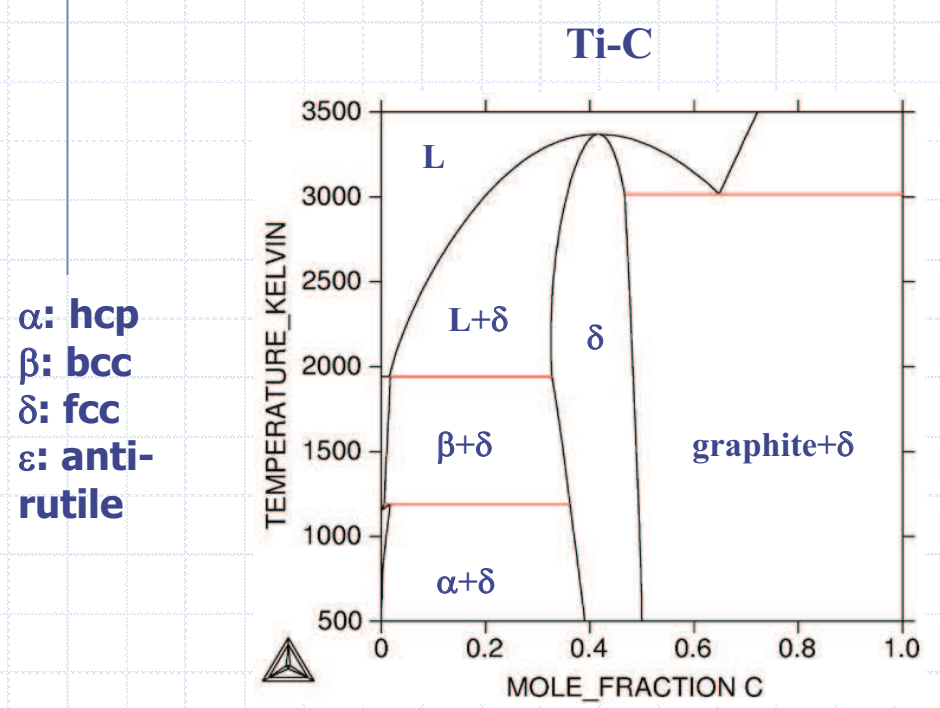

K. Frisk et al., Calphad (2004)
Ti-N

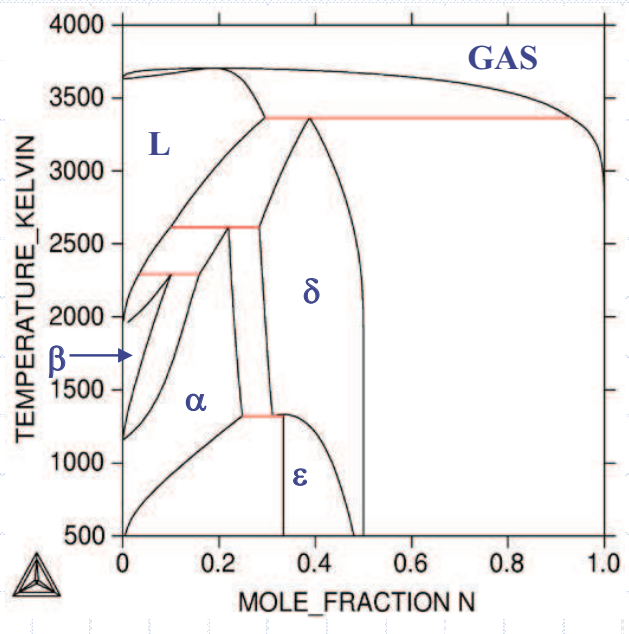

S. Jonsson Z. Metallkunde (1996) 


\section{Vacancy-vacancy interactions}

Cluster expansion: $\quad E(\sigma)=\sum_{f} D_{f} J_{f} \bar{\Pi}_{f}(\sigma)$
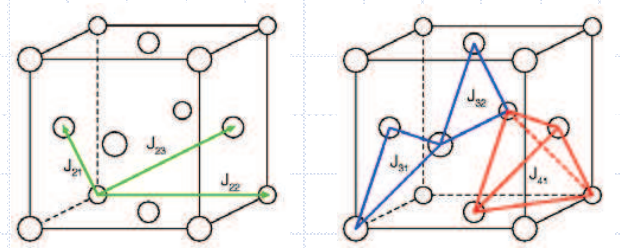

Theoretical methods:

Connolly-Williams method (CWM) Generalized perturbation method (SGPM) Vacancies prefer $3^{\text {rd }}$ nearest neighbor distance, $\langle 1,1 / 2,1 / 2\rangle$.

TABLE I. Effective carbon-vacancy interactions in $\mathrm{TiC}_{x}$ (degrees Kelvin). See text for the definitions.

\begin{tabular}{lrrrrrr}
\hline \hline & $J_{21}$ & $J_{22}$ & $J_{23}$ & $J_{24}$ & $J_{25}$ & $J_{26}$ \\
\hline SGPM & 54 & 181 & -10 & -1 & -12 & 24 \\
CWM & 113 & 246 & -13 & -5 & & \\
\hline & $J_{27}$ & $J_{28}$ & $J_{31}$ & $J_{32}$ & $J_{41}$ & \\
\hline SGPM & 1 & -5 & -10 & 53 & -6 & \\
CWM & & & -33 & 64 & -6 & \\
\hline
\end{tabular}

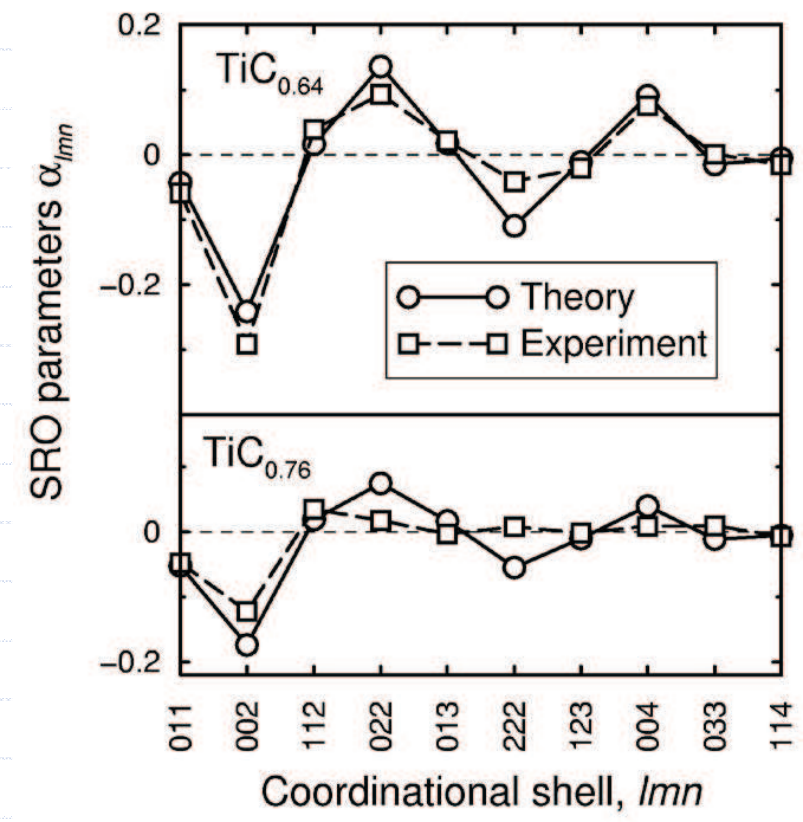

Experiment: T. Priem et al. (1989).

\section{Monte Carlo modeling of vacancy ordering}

Calculated phase diagram

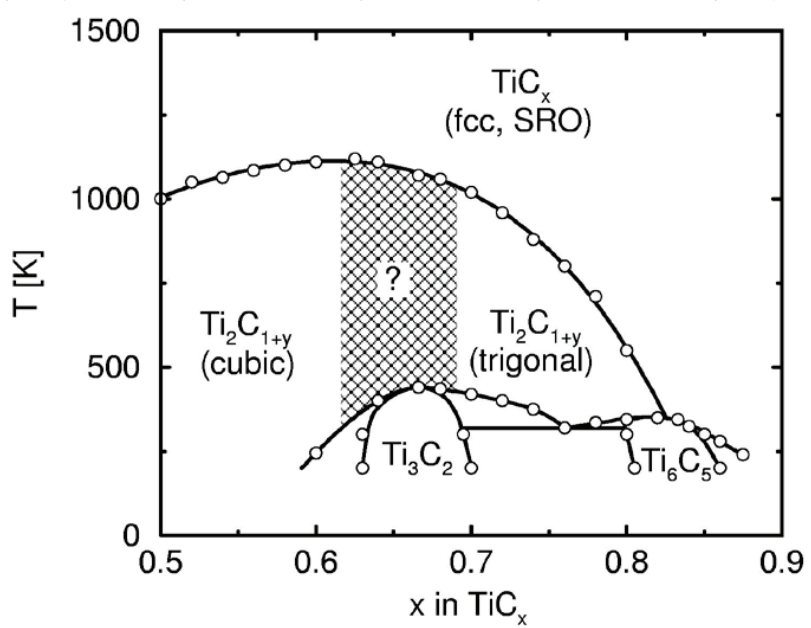

Order of vacancies in the (111) layers of $\mathrm{Ti}_{3} \mathrm{C}_{2}$ and $\mathrm{Ti}_{6} \mathrm{C}_{5}$ :

Ordering pattern

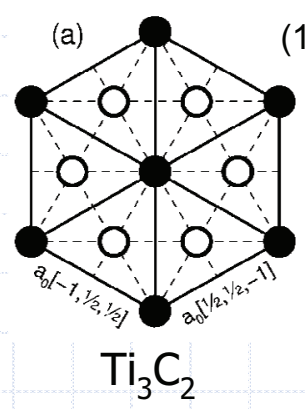

(111) (b)

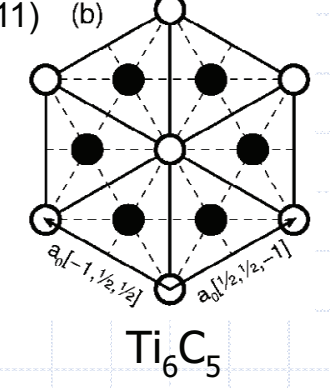

Stacking between layers

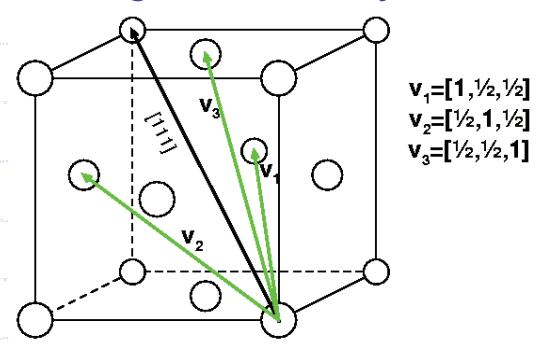

P. Korzhavyi et al., Phys. Rev. Lett. 88, 15505 (2002).

\section{Monte Carlo}

- Simulation box: 32×32×32 (32768 carbon sites)

- 5000 time steps per site 


\section{MC simulation of the ground state structures in $\mathrm{TiN}_{\mathrm{x}}$}

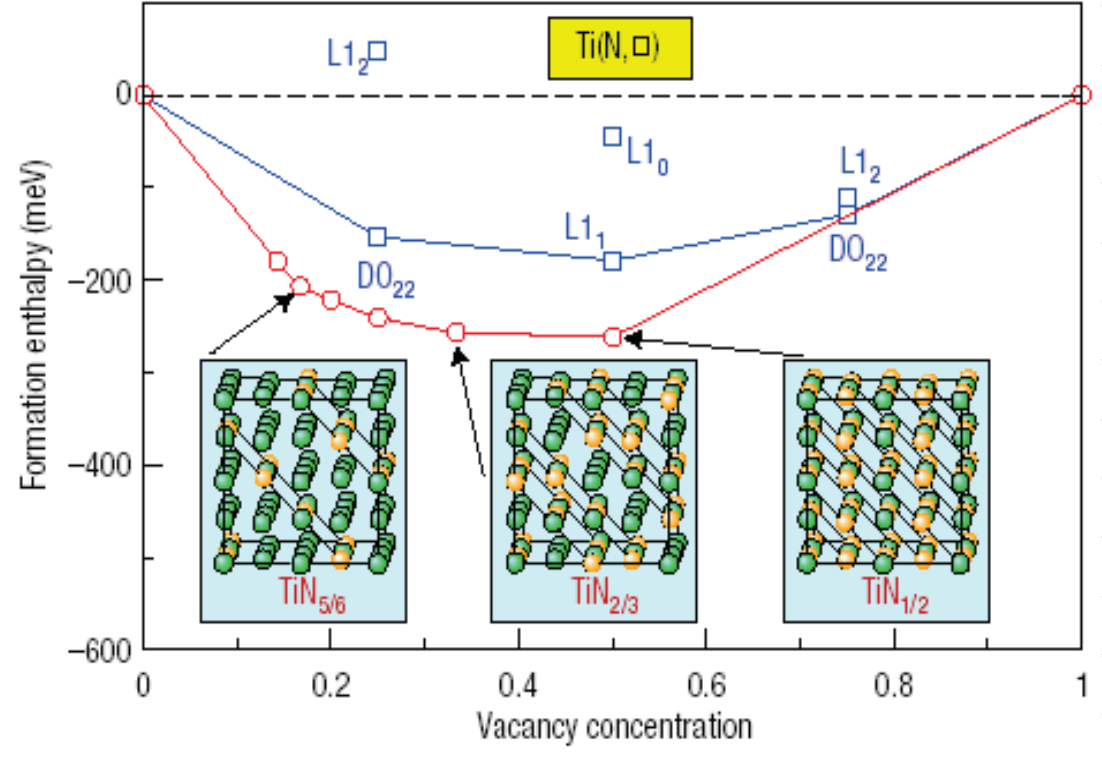

GUS L. W. HART, VOLKER BLUM, MICHAEL J. WALORSKI AND ALEX ZUNGER (Nature Materials, 2005)
- Ground state ordering is slightly different from $\mathrm{TiC}_{\mathrm{x}}$.

- Stacking of $(011)_{B 1}$ planes instead of $(111)_{B 1}$.

- All structures can be described within a 24 site unit cell (5 sublattices).

- Calculate the total energy of the structures and compare with the previous model.

\section{\begin{tabular}{|l|l|l|}
\hline Slide 27 & Theoretical data for assessments \\
\hline
\end{tabular}}

\section{CEF models for Ti-TiC-TiN}

\section{TiC-type}
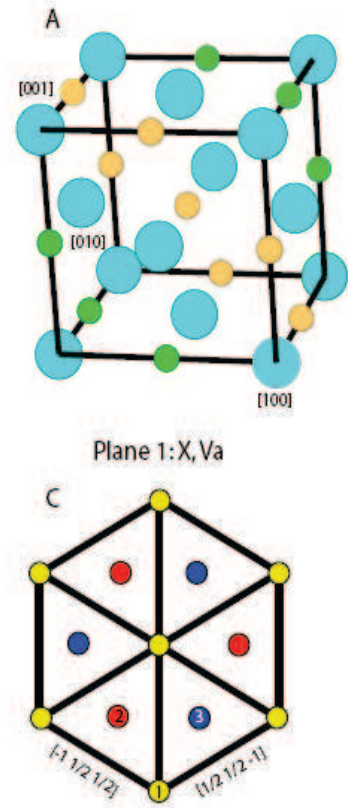
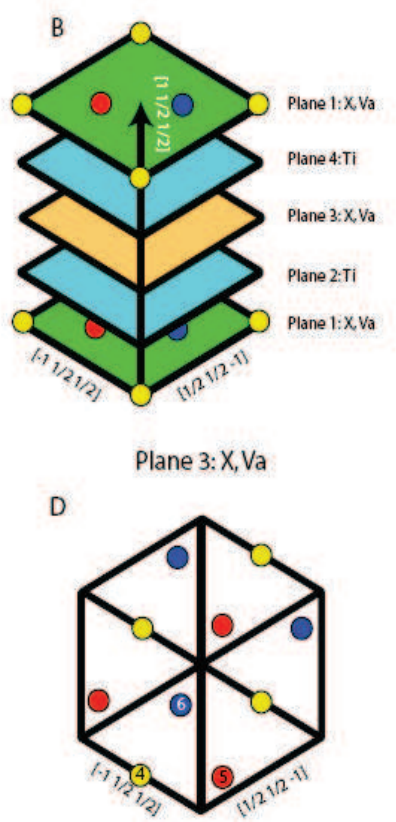

TiN-type
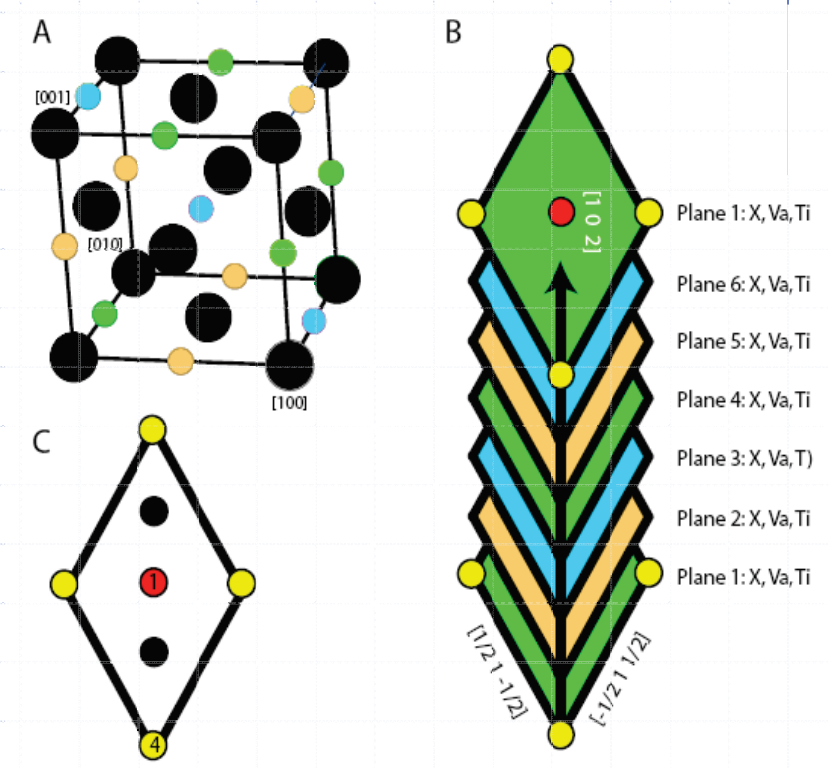


\section{Prediction of phase diagrams}
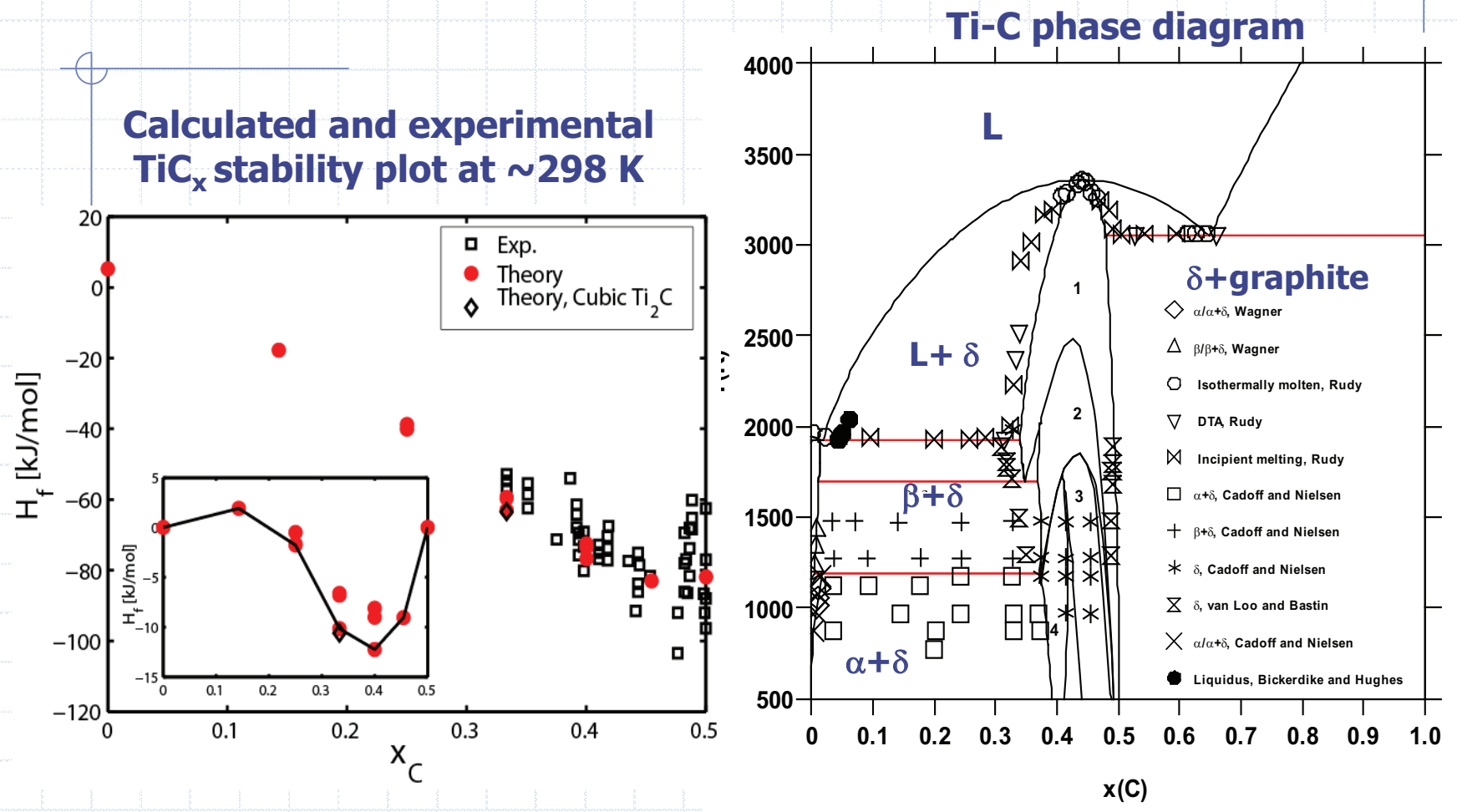

Slide 29

Theoretical data for assessments

$11 / 172010$

\section{Prediction of phase diagrams}

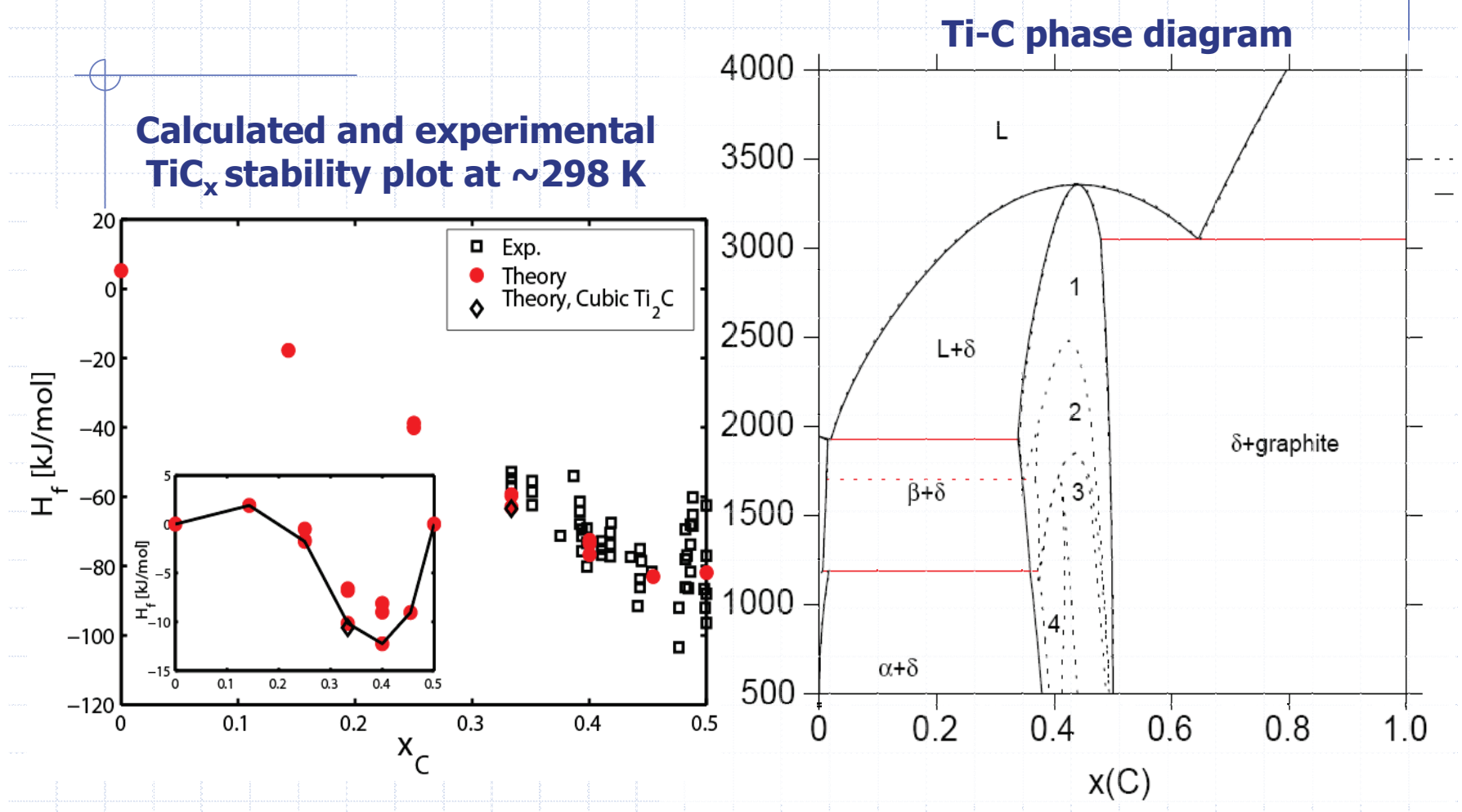




\section{Prediction of ternary phase diagrams and short range order}

Ti-C-N at $1423 \mathrm{~K}$
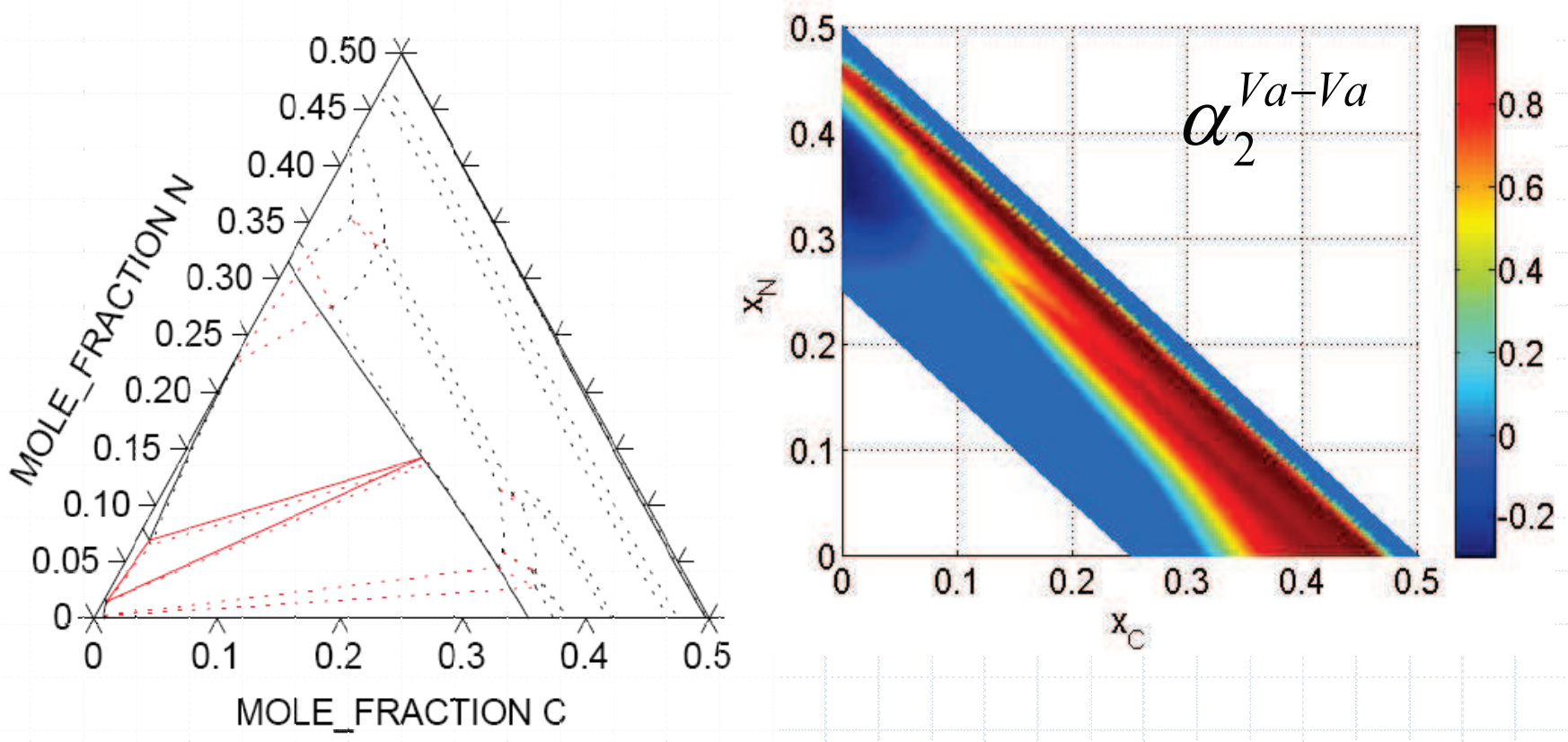